TITLE:

\title{
Method to Develop Pseudo Three- dimensional Dental Image from Dental Panoramic Radiograph
}

$\operatorname{AUTHOR}(S)$ :

Kuroda, Tomohiro; Kaga, Tetsuro; Azuma, Hiroko; Yagi, Masakazu; Kuroda, Yoshihiro; Imura, Masataka; Oshiro, Osamu; Takada, Kenji

\section{CITATION:}

Kuroda, Tomohiro ... [et al]. Method to Develop Pseudo Three-dimensional Dental Image from Dental Panoramic Radiograph. Biocybernetics and Biomedical Engineering 2011, 31(2): 59-70

\section{ISSUE DATE:}

2011-01

URL:

http://hdl.handle.net/2433/197321

\section{RIGHT:}

(c) 2011 Nacz Institute of Biocybernetics and Biomedical Engineering. NOTICE: this is the author's version of a work that was accepted for publication in Biocybernetics and Biomedical Engineering. Changes resulting from the publishing process, such as peer review, editing, corrections, structural formatting, and other quality control mechanisms may not be reflected in this document. Changes may have been made to this work since it was submitted for publication. A definitive version was subsequently published in Biocybernetics and Biomedical Engineering, 31(2), 2011,

doi:10.1016/S0208-5216(11)70011-0; This is not the published version. Please cite only the published version.; この論文 は出版社版でありません。引用の際には出版社版をご確認ご利用ください。 


\title{
METHOD TO DEVELOP PSEUDO THREE-DIMENSIONAL DENTAL IMAGE FROM DENTAL PANORAMIC RADIOGRAPH
}

\author{
Tomohiro Kuroda ${ }^{1}$, Tetsuro Kaga ${ }^{2}$, Hiroko Azuma ${ }^{3}$, Masakazu Yagi ${ }^{3,4}$, \\ Yoshihiro Kuroda $^{2}$, Masataka Imura ${ }^{2}$, Osamu Oshiro ${ }^{2}$, Kenji Takada ${ }^{3,4}$ \\ ${ }^{1}$ Department of Medical Informatics, Kyoto University Hospital, Kyoto, Japan \\ ${ }^{2}$ Graduate School of Engineering Science, Osaka University, Toyonaka, Japan \\ ${ }^{3}$ Graduate School of Dentistry, Osaka University, Suita, Japan \\ ${ }^{4}$ The Center for Advanced Medical Engineering and Informatics, Osaka University, Suita, \\ Japan
}

Abstract-Although three-dimensional image can be a powerful tool for dentists to explain treatments to patients, obtaining of three-dimensional image of teeth in general dental clinics is difficult. This paper proposed a method to develop pseudo three-dimensional dental image from conventional dental panoramic radiograph and dental impression. The method estimates imaging parameters of given panoramic radiograph through comparison with dental cast, and re-projects the radiograph into three-dimensional space. The developed pseudo threedimensional image gives clear impression of the patient's dental condition.

Keywords; dental panoramic radiograph; dental impression; three-dimensional image development

\section{INTRODUCTION}

Recent rapid advancement of medical imaging technologies and computational technologies provides enormous advantages for clinical activities such as informed consent as well as clinical diagnosis. Especially three-dimensional volumetric images produced by X-ray computed tomography (X-CT) or magnetic resonance imaging (MRI) are indispensable tools for modern clinical activities. However, MRI is not applicable to imaging bones and teeth due to its poor spatial resolutions [1], X-CT is not applicable for imaging head, region surrounding brain, for fear of radiation exposure [2,3]. Although low-dose dental cone-beam CT is emerging [4,5], its high expense prevents spreading among dental clinics, and its limited range of imaging limits applicable cases. Thus, general dental clinics are not using three-dimensional images in their daily clinical activities.

This paper proposes a new method to develop pseudo three-dimensional dental image from general information resources used in dental clinics, that is, panoramic radiograph and dental impression.

\section{RESOURCES}

This section presents resources to develop pseudo three-dimensional dental image.

The proposed method utilizes data resources obtained in general clinical activities, that is, dental impression and dental panoramic radiograph. The following sections will present utilized method to obtain three-dimensional surface data from dental impression, and basics of dental panoramic radiography. 


\subsection{Dental Impression}

In general clinical processes, dentists or dental technicians take dental impression and make dental cast of patients' mouth to represent their dentition. Dental impression [6] is a "negative" of those teeth or gum for such casting. To obtain dental impression, dentists or dental technician presses viscous liquid, most often alginate sodium, filled in impression tray, and wait till the liquid becomes elastic solid to hold shape of teeth and gum.

Three-dimensional digitizer can obtain three-dimensional surface dataset of teeth and gum from dental cast. Most of digitizers available on today market measure shape of target object using the light section method shown in figure 1. The method projects slit-light onto target object and shoot it from other direction, and work out shape by stereo measurement theory [7]. Although the method requires mechanism to scan slit-light, it can measure shape of target object regardless of surface color or texture. As dental cast doesn't have any referable surface texture, active stereo measurement including light section method is preferable.

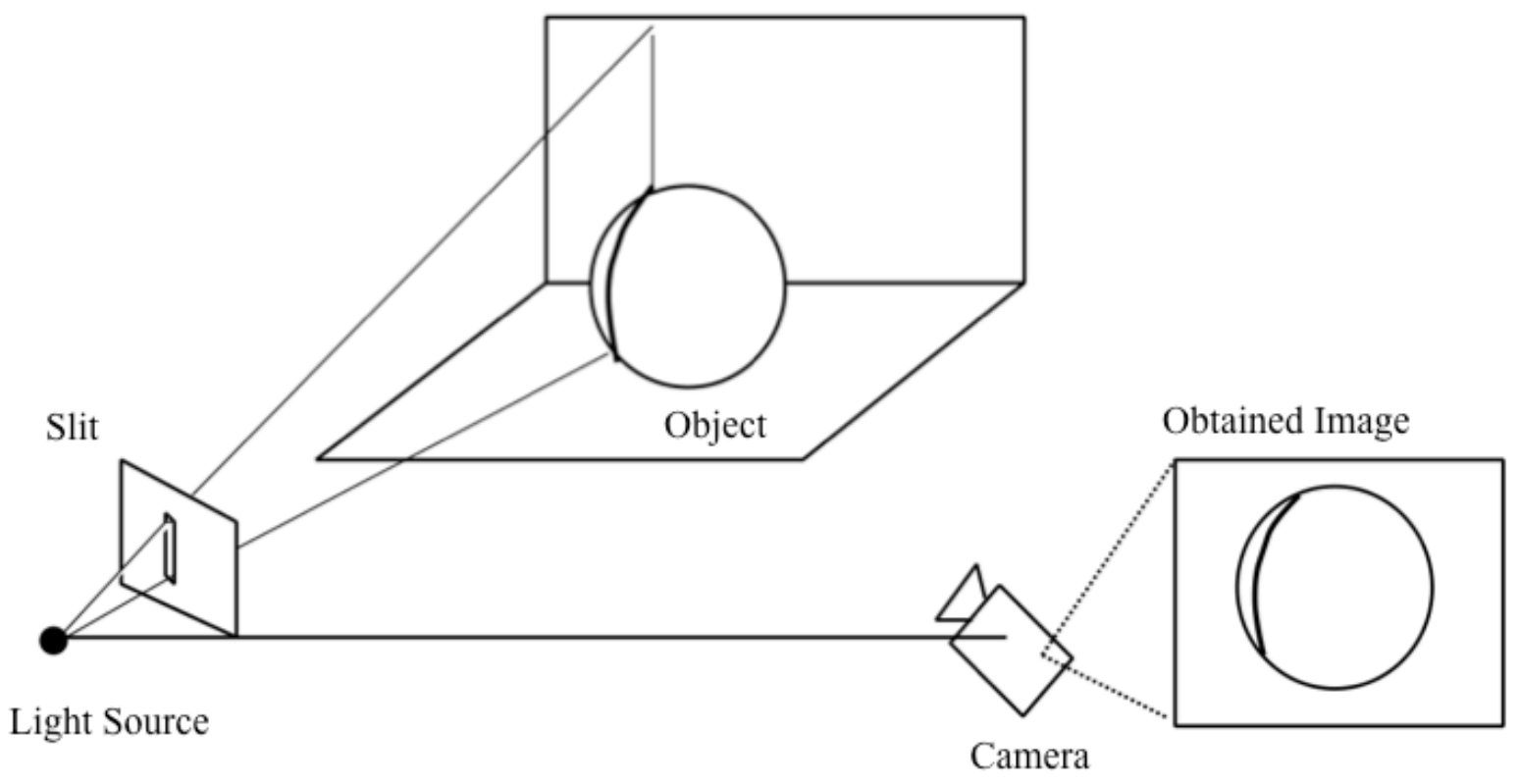

Figure 1. Schematic diagram of light section method

\subsection{Panoramic Radiography}

Panoramic radiography [8] is an imaging method for teeth based on principles of tomography.

Figure 2 shows mechanism of most basic panoramic radiography named pantomography. The method rotates radiation source and film around a certain rotation axis $O_{l}$. As imaging range, shaded area in figure 2, becomes Baumkuchen-like shape, whole dental arch won't be projected precisely. Front teeth away from radiation source appear to be narrow, and back teeth close to radiation source appear to be wide as shown in figure 3. To avoid such distortion, several more complex panoramic radiographies to fit imaging range with dental arch, such as panorex radiography, orthpantomography, and elipsopantomography, have been developed. 


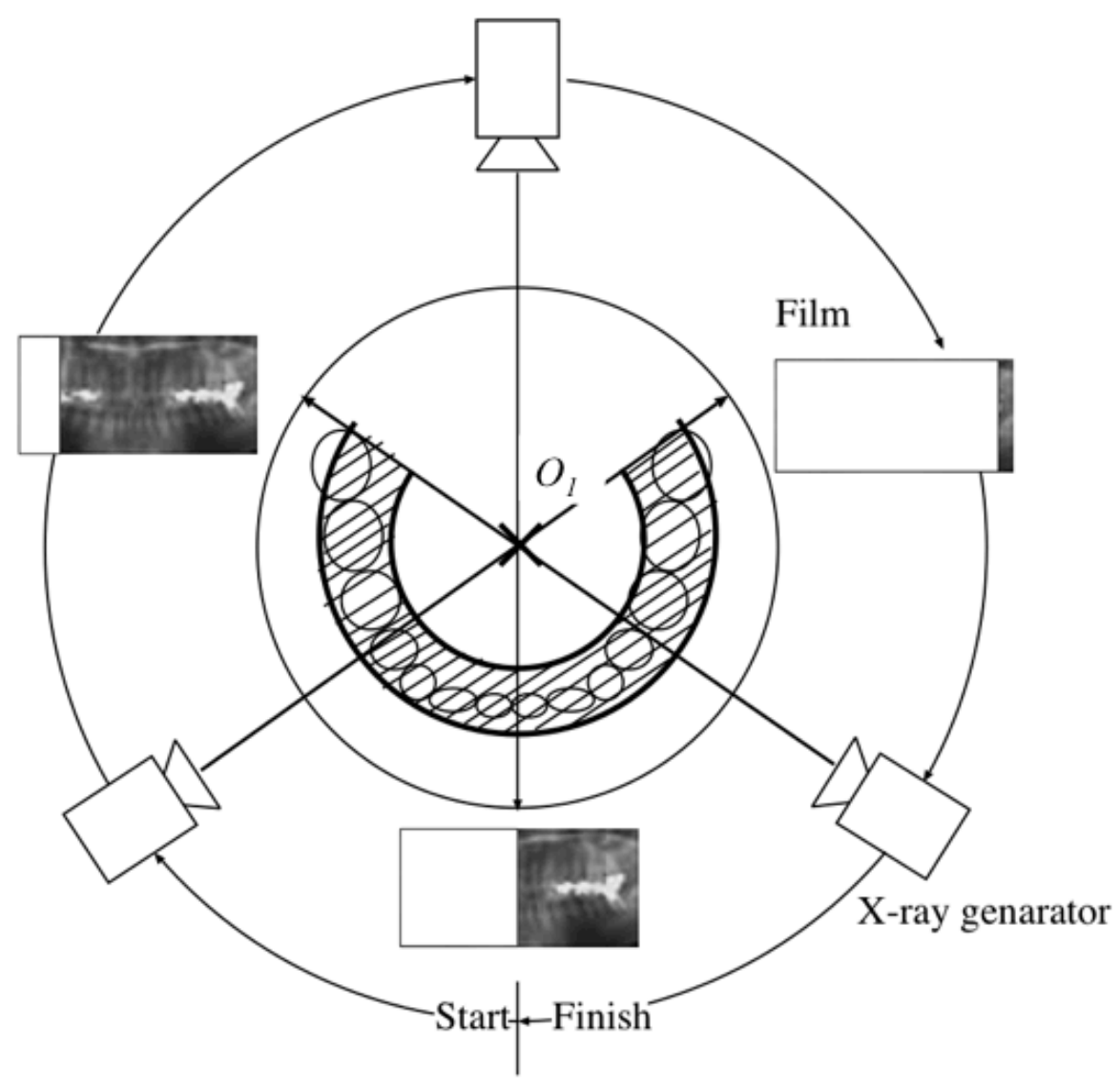

Figure 2. Mechanism of pantomography

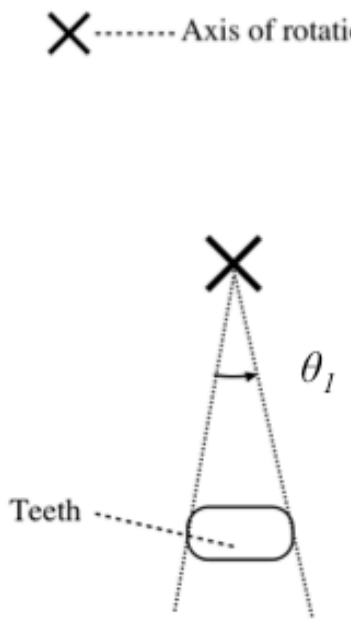

A

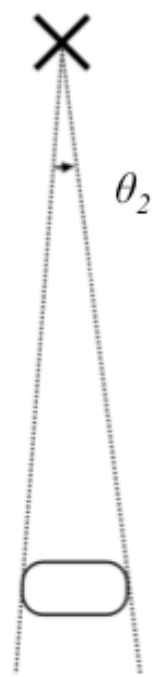

B

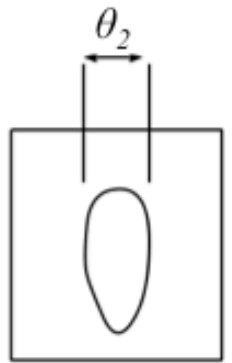

B

Figure 3. Distortion caused by pantomography 


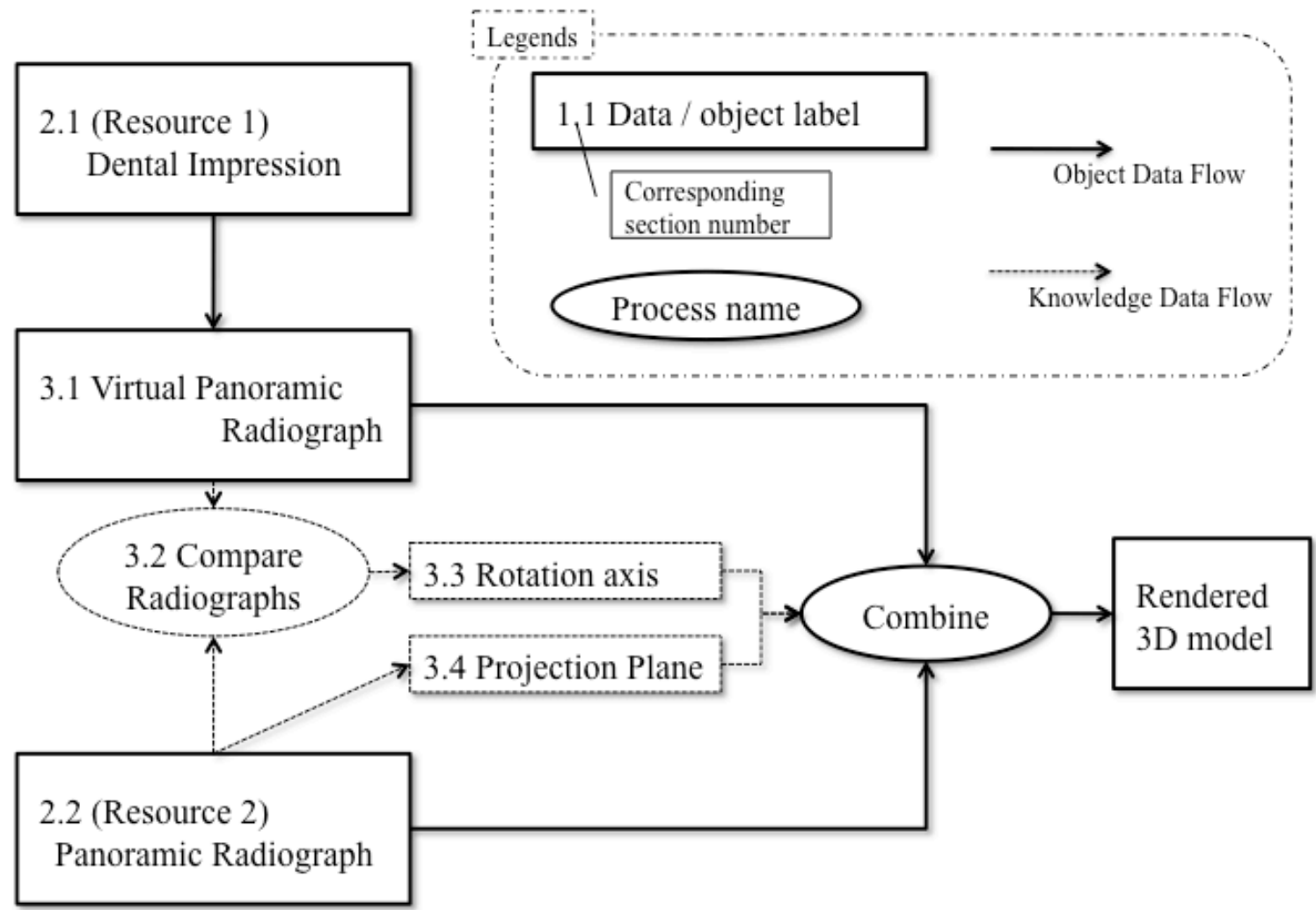

Figure 4. Process flow of proposed method

Although the panoramic radiograph provides transmission image of the whole teeth and mouth, it won't give intuitive impression for non-professionals such as patient. The authors thought a panoramic radiogram arranged along original dental arch might present better idea of dentition for patients.

Figure 4 shows the process flow of the proposed generation method. The method estimates rotation axis and tomographic plane by comparing panoramic radiograph and virtual panoramic radiograph generated from three-dimensional surface dataset of teeth and gum obtained via dental cast.

The following sections present details.

\subsection{Generating virtual panoramic radiograph}

To compare two datasets of different dimensions, reduction of dimensions of dataset with higher dimension is indispensable.

The proposed method develops virtual panoramic radiographs from three-dimensional surface dataset with various imaging parameters, and finds the one which is most similar to the obtained real radiograph to define imaging parameters.

Intensity of each pixel of the radiograph expresses absorbed dose. The authors introduce quite simplified assumption that the intensity that expresses absorbed dose is proportional to thickness of the teeth. Therefore, the intensity of certain pixel can be worked out from $T_{1}, T_{2}$, and $T_{3}$ in figure 5 , when one virtual camera placed at the rotation axis and another camera placed $T_{3}$ away. 


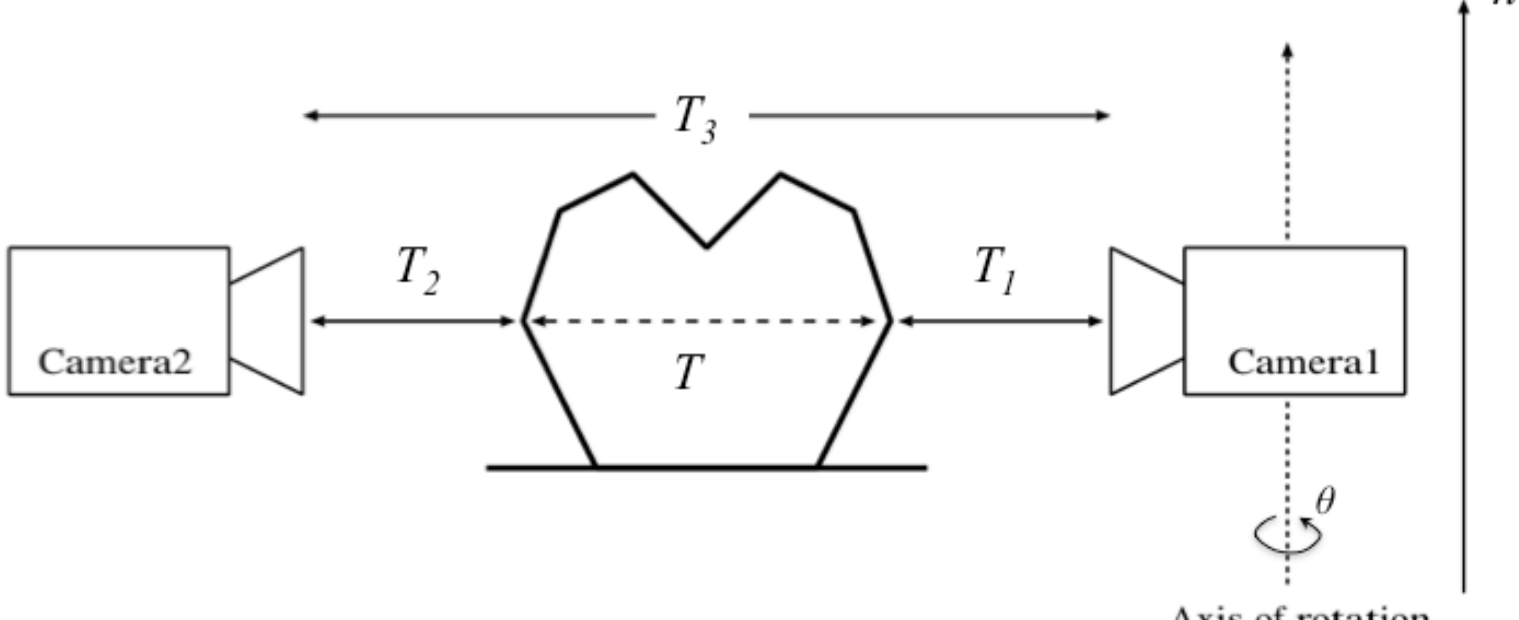

Figure 5. Measuring thickness of teeth

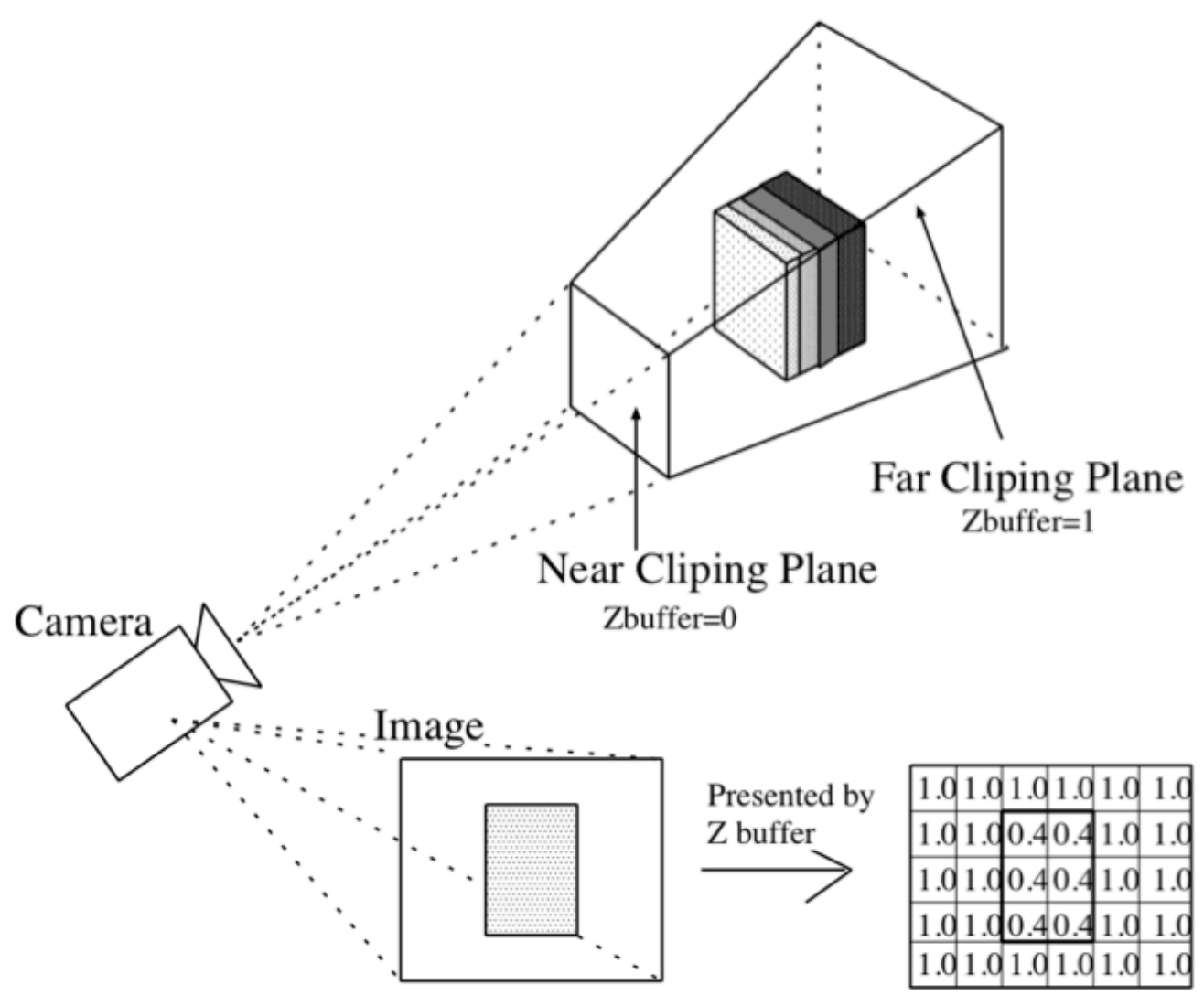

Figure 6. $\quad$ Z buffer

Distance from the camera to the surface of teeth, $T_{1}$ and $T_{2}$, is obtained via $\mathrm{Z}$ buffer. As shown in figure $6, \mathrm{Z}$ buffer stores depth of each pixel from the camera under parallel projection; Zero indicates position of near clipping plane and one indicates position of far clipping plane.

Finally, calculated $T$ s along vertical line including image center in each snapshot are placed along rotating angle to generate the virtual panoramic radiograph. 


\subsection{Comparing virtual and real panoramic radiograph}

To evaluate resemblance of the generated virtual panoramic radiograph and the real panoramic radiograph, the method compares width of each tooth on the two images. As discussed in section 2.2, the image of each tooth represents distance from the rotating center to each tooth. Therefore, if the rotating axes of two panoramic radiographs match, the width of each tooth should match, too.

In panoramic radiography, images of front teeth are overlapped blurred images of cervical bones, and image of molars of each side tends to be overlapped each other due to distortion. Therefore, extracting of each tooth independently is quite difficult. Thus, the authors defined evaluation value $C_{1}$ as shown in (1), considering ratio of molars within dental arch.

$$
C_{1}=\left|\frac{a}{d}-\frac{a^{\prime}}{d^{\prime}}\right|+\left|\frac{b}{d}-\frac{b^{\prime}}{d^{\prime}}\right|+\left|\frac{c}{d}-\frac{c^{\prime}}{d^{\prime}}\right|
$$

Here, parameters in (1) are as shown in figure 7.
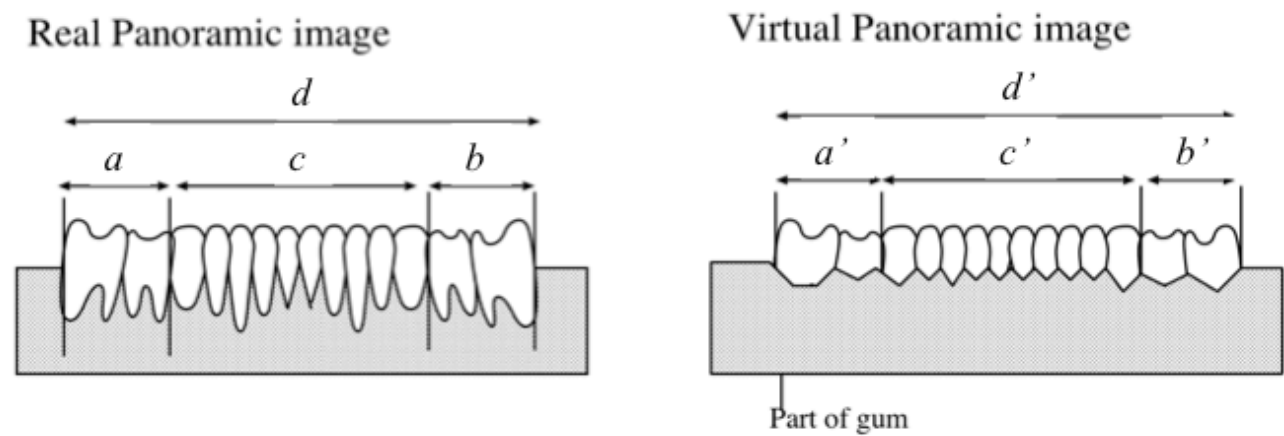

Figure 7. Comparing parameters of panoramic radiographs

The parameters, widths of sets of teeth, are worked out by extracting interdental gaps. As intensity changes quite rapidly around the gaps, lateral derivative is utilized. Figure 8 shows the method of extracting the gaps. The method binarizes lateral derivation with a certain threshold, and produces the histogram along the horizontal line. The higher histogram represents the gaps. Of course, dental prosthesis harms lateral derivative of panoramic radiograph as image of prosthesis has generally higher intensity. Therefore, image of prosthesis must be eliminated manually before generating the binary image. 
Real panoramic image

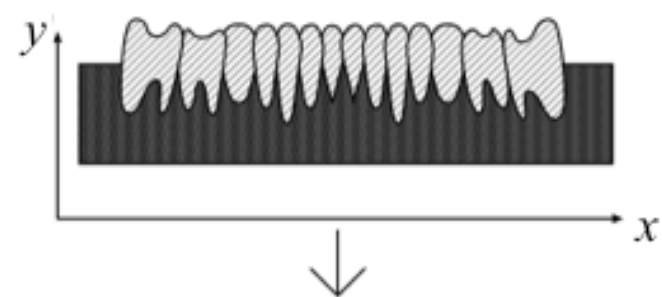

Laterally derivated

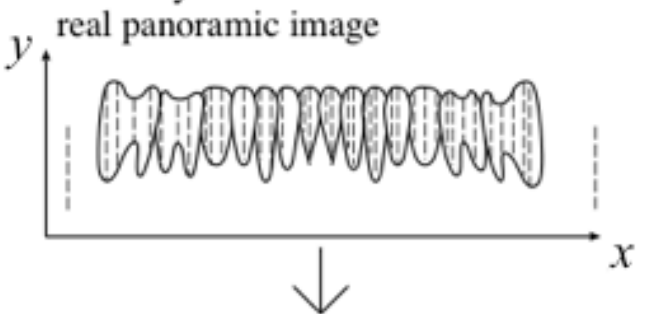

Binarized

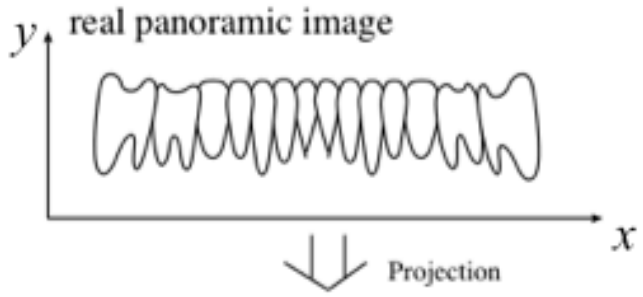

Counted number of pixel

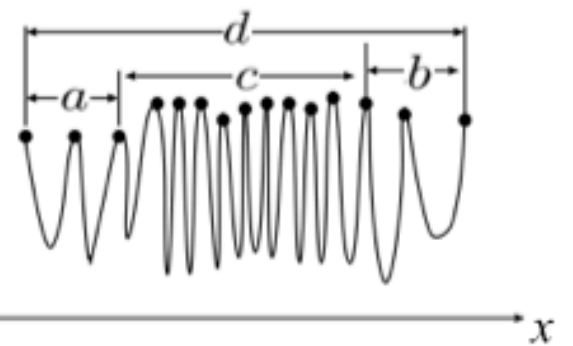

Virtual panoramic image

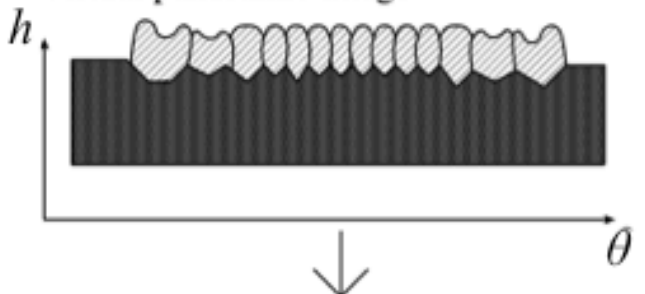

Laterally derivated virtual panoramic image $h$

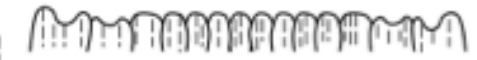

Binarized

$h$, virtual panoramic image
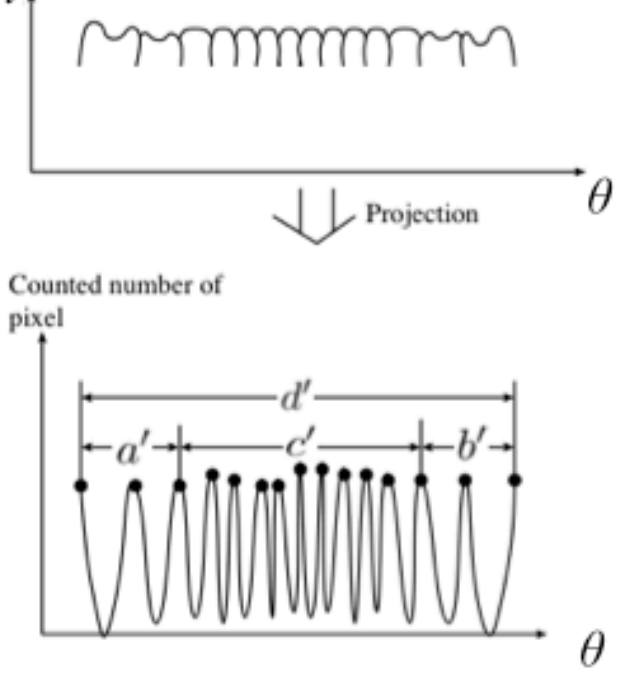

•.......... Local Maximum

Figure 8. Extraction of interdental gaps 


\subsection{Finding rotation axis}

The method of finding the rotation axis is as follows. Fist of all, the method places rotation axis candidates in a certain regular distance $e$ as shown in figure12, and generates the virtual panoramic radiograph for each candidates. The generated images are compared with the real one using evaluation value $C_{1}$, and the best matching virtual panoramic radiograph is selected. Consequently, the method places the rotation axis in half distance $e / 2$ as shown in figure12, and performs the matching process again. The method iterates the matching process until the distance between candidates become smaller than resolution of range finder to measure dental cast.

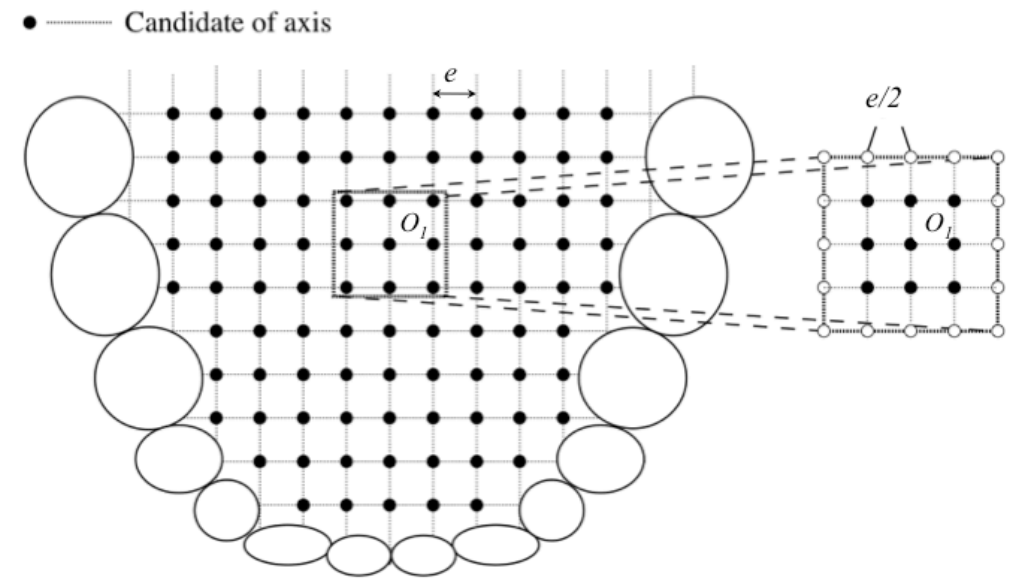

Figure 9. Finding rotation axis

\subsection{Defining projection plane}

As the tomographic plane of an original panoramic radiograph should exist within dental arch, the method defines the projection plane as the center of dental arch. The center of each tooth can be obtained easily via process of estimating of thickness of each tooth discussed in 3.1. The method defines the projection plane as a plane connecting obtained centers as shown in figure 10 .
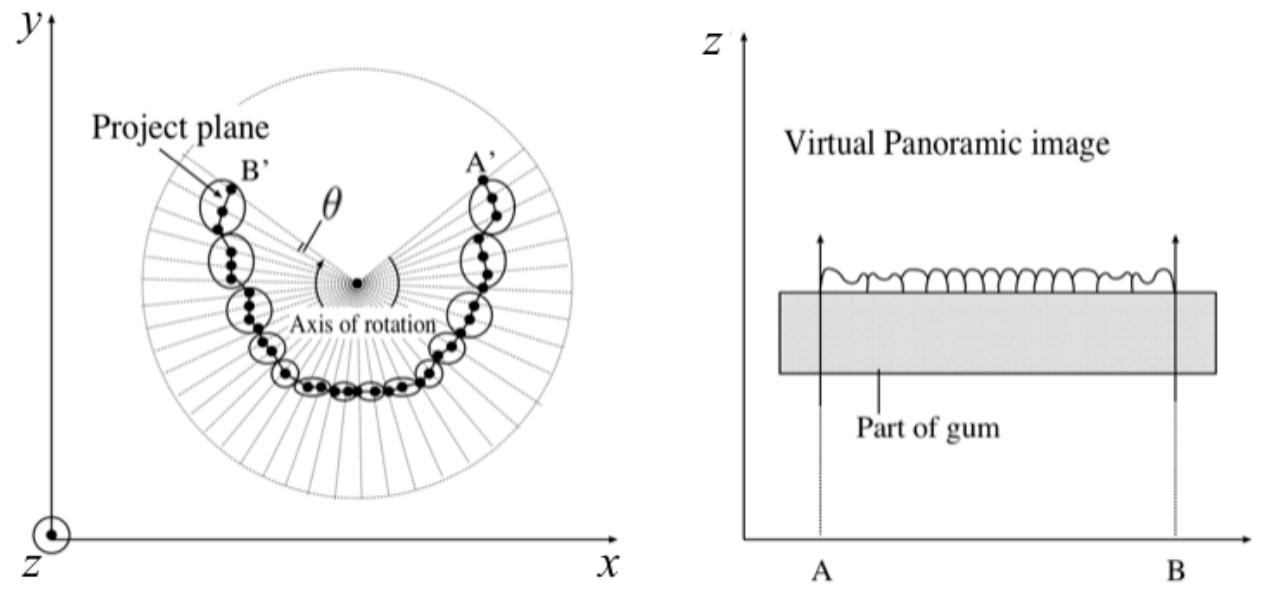

Figure 10. Defining projection plane

Finally, the obtained real panoramic radiograph is projected onto generated surface by the conventional texture mapping technique. 


\section{Evaluation}

The developed method is applied to a dataset obtained from dental model for training.

Target dental model is I2D-400C (Nisshin), dental impression material is Aroma Find DF3 (GC), and plaster is New Plastone (GC).

The developed plaster model is digitized by SURFLACER (UNISN), a digitizer based on light section method. Resolution of the digitizer is $0.25 \mathrm{~mm}$.

As the target dental training model is made of epoxy resin, absorbed dose is not enough to get radiograph via general dental radiography. To increase the absorbed dose, surface of each tooth model is covered by nickel via chrome plating. The panoramic radiograph of the model is obtained by smx-100 (Shimadzu). The tube voltage was $70 \mathrm{kV}$ and tube current was $10 \mathrm{~mA}$. The target model was set to fit the midline of model to the rotation axis and to fit the occlusal plane to the horizontal plane. The obtained image was digitized with GT-X970 (Epson) film scanner.

The two datasets are compared using the interdental gaps as discussed in 3.2.

Consequently, the authors tried to find the rotation axis. As the model placed to fit midline of the dental model to the rotation axis, the authors limits search area only along midline. Figure 11 shows search space of initial iteration. Distances between candidate points are $8 \mathrm{~mm}$. Figure 12 shows evaluation value $C_{l}$. The estimated rotation axis was at $y=-10.25 \mathrm{~mm}$.

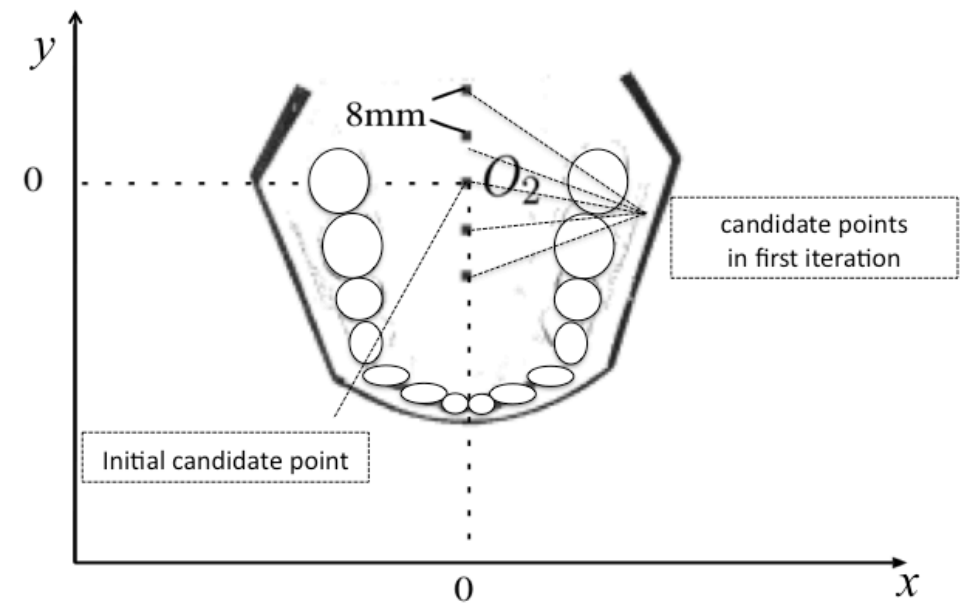

Figure 11. Search space and initial candidate points of rotation axis

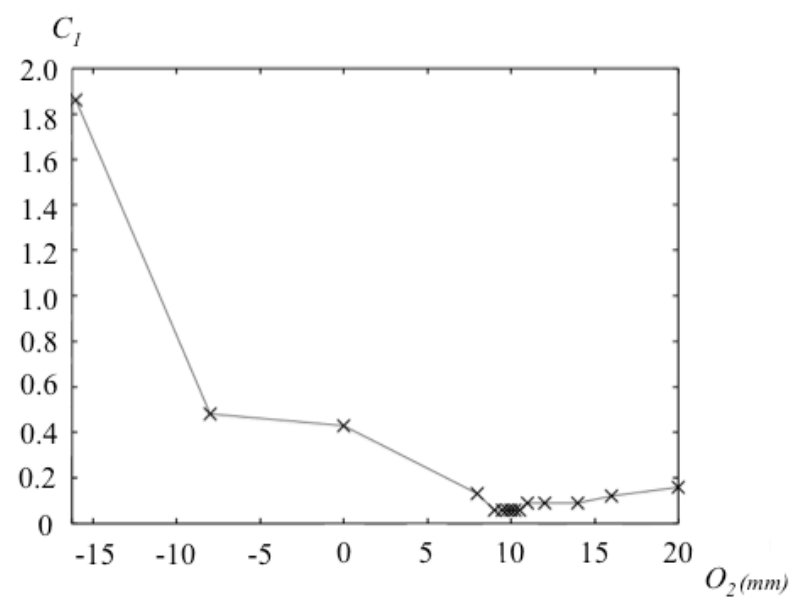

\begin{tabular}{|c|c|}
\hline $\begin{array}{l}\text { Seeking } \\
\text { distance }(\mathrm{mm})\end{array}$ & Seeking area(mm) \\
\hline 8.0 & $-16.0 \sim 16.0$ \\
\hline 4.0 & $12.0 \sim 20.0$ \\
\hline 2.0 & $10.0 \sim 14.0$ \\
\hline 1.0 & $9.0 \sim 11.0$ \\
\hline 0.5 & $9.5 \sim 10.5$ \\
\hline 0.25 & $9.75 \sim 10.25$ \\
\hline
\end{tabular}

Figure 12. Profile of evaluation value $C_{l}$ 
Finally, the projection plane was extracted and the original panoramic radiograph superimposed into the three-dimensional surface image. Figure 13 shows the resulting pseudo three-dimensional dental image.

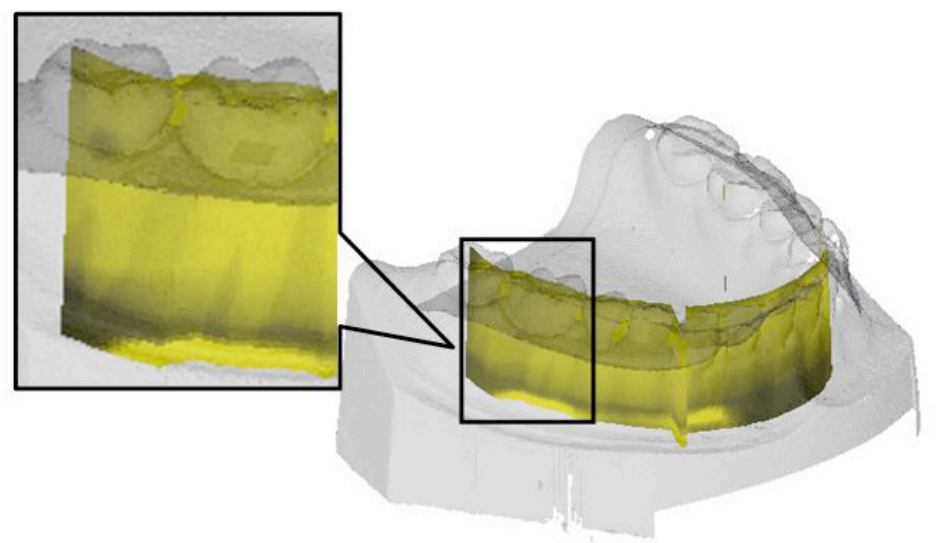

Figure 13. Generated pseudo three-dimensional dental image

\section{Discussion}

The generated pseudo three-dimensional image gives intuitive impression of dental condition as radiographic image including tooth roots within three-dimensional surface data. Although the generated image has poor contrast, it won't harm advantage of the proposed method as the poor contrast is derived from poor radiation absorption of target phantom, chrome plated epoxy resin teeth.

Some dentists including authors find advantage of the generated image to share impression of depth of dental caries before starting the treatment. Series of datasets of a certain patient before and after treatment visualized in the proposed method may give better understandings to effect of the treatment. Especially, such image sequence can be powerful tool especially in orthodontics, not only for informed consent but also for education.

This paper defines projection plane as a center of dental arch to visualize radiograph within surface of teeth. However, as original tomographic plane is cylinder, simple fitting of cylindrical projection plane to dental arch may generate a good enough pseudo threedimensional image. The difference of given impression between simple cylinder projection plane and the proposed method remains open question.

As this paper assumes that the occlusal plane of target dentition is perpendicular to the rotation axis of panoramic radiograph, the search space is limited to a two-dimensional plane. However, the occlusal plane cannot be always perpendicular to the rotation axis due to wide variation of human head. To make the proposed method applicable for general clinics, to expand search space to additional dimension, most probably pitching, or to develop suitable guides to set the occlusal plane to preferable orientation, is indispensable.

This paper handles most basic panoramic radiograph, pantomograph. Although, more complex methods, such as panorex radiography and orthpantomography, have multiple rotation axes to fit imaging area to dental arch, the basic approach of the proposed method must be applicable to estimates those multiple axis. Of course, more research is required to prove this estimation.

In the future, some vender of dental radiography may provide imaging parameter of certain panoramic radiography. Even if imaging parameters are given, certain computation to fit radiography with surface model is still unavoidable. However, the given parameters contribute to drastic reduction of search space. 


\section{CONCLUSION}

This research proposed and developed a method to generate pseudo three-dimensional dental image from conventional panoramic radiograph.

The proposed method provides powerful tool for daily clinical activities such as informed consent, because the generated pseudo three-dimensional image gives intuitive impression of dental condition for patients. Additionally, as the method won't require any additional imaging devices to generate the pseudo three-dimensional image, it is applicable for general dental clinics. The authors hope the proposed method become available for general dental clinics to smoothen clinical activities.

\section{ACKNOWLEDGEMENTS}

The authors would like to thank Dr. Kiyohito Yanagi and Dr. Miho Torii of Yanagi Orthodontic Clinic, Mr. Hirohiko Kondo of Kondogikenkogyo corp., Prof. Kenji Matsumoto and Prof. Masao Tanaka of Graduate School of Engineering Science, and Dr. Yuri Yamamoto of Graduate School of Dentistry, Osaka University for their precious support to perform this research project.

This research is partly funded by Osaka University Global COE program, a center of excellence for in-silico medicine oriented worldwide platform project.

\section{REFERENCES}

1. K. Yamada, E.R. Wisner, J.S. de Ropp, R.A. LeCouteur, and L.D. Tripp, "Technical parameters affecting image characteristics in in vivo MR microscopy of the mouse," Veterinary Radiology and Ultrasound, Vol.43, No.6, pp.518-527, 2002.

2. C.H. McCollough, and F.E. Zlink, "Performance evaluation of a multi slice CT system,” Medical Physics, Vol.26, No.11, pp.2223-2230, 1999.

3. L.N. Rothenberg, and K.S. Pentlow, "CT dosimetry and radiation safety in Medical CT and Ultasound: Current Technology and Applications, L.W. Goldman and J.B. Fowlkes, Eds. Madison, WI: Advanced Medical Publising, 1995, pp. 519-553.

4. W.C. Scarfe, A.G.Farman, and P.Sukovic, "Clinical applications of bone-beam computed tomography in dentl practice," Journal Canadian Dental Association, Vol.72, No.1, pp. 75-80, 2006.

5. M.E. Guerreo, R. Jacobs, M. Loubele, F. Schutyser, P. Suetens, and D. van Steenberghe, "State-of-the-art on cone beam CT imaging for preoperative planning of implant placement,", Clinical Oral Investigations, Vo.10, No.1, pp. 1-7, 2006.

6. G.E. Gordon G.H. Johnson, and D.G. Drennon, "The effect of tray selection on the accuracy of elastmeric impression materials," The journal of prosthetic dentistry, Vol. 63, No. 1, pp.12-15, 1990.

7. S. Inokuchi and K. Sato, Three-dimensional image based measurement, Tokyo : Shokodo, 1990, Japanese.

8. O.E. Langland, R.P. Langlais, and C.R. Morris, Priciples and practice of panoramic radiology: including intraoral raidographic interpretation, Philadelhia : Saunders, 1982. 